\title{
Raman Spectroscopy as a Powerful Technique in the Characterization of Ferrofluids
}

\author{
Joel C. Rubim*, Marcelo H. Sousa, Jean C. O. Silva, and Francisco A. Tourinho \\ Instituto de Química da Universidade de Brasília \\ CP 04478, 70919-970, Brasília, Brazil
}

Received on 29 January, 2001

\begin{abstract}
Raman spectroscopy has been used to get some insight into the chemical composition and structure of magnetic fluids based on ferrites. The inner as well as the surface structure of Fe-maghemite and Zn-maghemite have characterized by Raman spectroscopy. It has been shown that different chemical species are present on the maghemite surface by changing the laser excitation energy. Maghemites modified by the adsorption of aspartic and glutamic acids as well as those modified by the adsorption of methylene blue(MB) have also been investigated by Raman spectroscopy. It has been shown that while FTIR (Fourier transform infrared) spectroscopy gives almost no information regarding to the surface species, Raman spectroscopy in and off resonance gives suitable information regarding to the adsorbate structure and bonding. The Raman spectroscopy study of the modified maghemites have shown that the organic acids adsorb on the maghemite surfarce as glutamate and aspartate chemically bounded to Fe(III) ions on the maghemite surface, and that $\mathrm{MB}$, a cation, adsorbs on the maghemite surface as a monomer by ion pair formation with coadsorbed nitrate.
\end{abstract}

\section{Introduction}

The advances in the field of nanomaterials ask for experimental techniques that can give information regarding to the internal structure of the nanoparticles as well as to their surface structure, since some properties presented by these materials are strongly related to surface structure and properties. Porous silicon and magnetic fluids are good examples of such kind of nanomaterials [1-5].

The development of Raman instrumentation having high light throughput, equipped with high performance CCD detectors, the use of optical microscopes to focus the laser on the sample and collect the scattered radiation, and the notch filter technology caused a tremendous increase in the use of Raman spectroscopy as an analytical tool. For instance, molecules adsorbed on surfaces at monolayer levels can be detected by Raman spectroscopy even if the system does not present surface-enhanced Raman characteristics [6,7]. Furthermore, if the system is in solution and strongly absorbing, as is the case of magnetic ferrofluids, the use of confocal spectroscopy makes possible the acquisition of good quality Raman spectra [8]. In this case, the use of a large amplification objective (x80) to focus the laser on the sample, a small silt width $(100 \mu \mathrm{m})$, and a digital pin hole generated at the CCD detector, guarantees a very small scattering volume avoiding re- absorption of the scattered light as well as reduces the strong flu- orescence background from the glass capillary walls.

Raman spectroscopy can also be seen as a vibronic spectroscopy, since it can give information regarding to both molecular aspects, the vibrational and electronic [9]. The works of Heller and collaborators [9-11] are excellent references where the Raman scattering phenomena, non resonant and resonant, are described in the time domain formalism. In their description once the laser photon interacts with the molecule a vibrational wave packet of the ground electronic state is pumped to the first excited state. If the photon energy is close to an allowed electronic transition, this wave packet will evolve in the excited state. After a finite time, the system relaxes and a Stokes shifted Raman photon is emitted. In this case, the vibrational modes associated to the chromophore structure, responsible for the electronic transition will be preferentially enhanced. In this sense, by just tuning the laser energy in a value corresponding to the absorption of a chromophore part of the investigated system their vibrational modes will be selectively enhanced.

In this paper we are going to drive our attention to the use of Raman spectroscopy in the study of chemically synthesized ferrofluids, or magnetic fluids, formed by the dispersion of nanosized ferrite $\left(\mathrm{M}(\mathrm{II}) \mathrm{Fe}_{3} \mathrm{O}_{4}, \mathrm{M}=\right.$ $\mathrm{Fe}, \mathrm{Zn}, \mathrm{Co}, \mathrm{Cu}, \mathrm{Mn}$, etc.) particles into an appropriate solvent $[4,5]$. This kind of sols, named as electric double layered ferrofluids (edl-ff) [5] present high surface areas and the modification of this surface by a suitable 
adsorbate can generate magnetic fluids with different properties, some of them of biomedical interest, known as biocompatible magnetic fluids [12].

In this work we will report the application of surface Raman spectroscopy in the characterization of non modified electric-double-layered-ferrofluids (edl$\mathrm{ff}$ ), on edl-ff modified by the adsorption of organic acids $\left(\mathrm{HO}_{2} \mathrm{CCH}_{2} \mathrm{CH}_{2} \mathrm{CHNH}_{2} \mathrm{CO}_{2} \mathrm{H}=\right.$ glutamic and $\mathrm{HO}_{2} \mathrm{CCH}_{2} \mathrm{CHNH}_{2} \mathrm{CO}_{2} \mathrm{H}=$ aspartic) as well as those modified by the adsorption of methylene blue (MB). These surface modifiers are very important in the preparation of biocompatible magnetic fluids and in the treatment of cancer by laser photodynamic therapy. Methylene Blue is known as a singlet molecular oxygen $\left({ }^{1} \mathrm{O}_{2}\right)$ generator $[13,14]$. Therefore it would be a good candidate for the treatment of cancer by laser photodynamic therapy [15]. On the other hand, as it happens to other photosensitive drugs, MB presents several undesired side effects [13]. In this sense the magnetic nanoparticle can be viewed as a local drug deliver that can be controlled by a magnetic field. In this work we will also discuss the effect of changing the laser excitation energy in the Raman spectra of the adsorbed MB as well as on the Raman spectra of the non modified edl-ff in order to show how different laser energies can excite different surface species and chromophores.

\section{Experimental}

In this work we have made use of edl-ff based on maghemite $\left(\gamma-\mathrm{Fe}_{2} \mathrm{O}_{3}\right)$ that were prepared according to a previously described procedure $[4,5]$. The Zn-edl-ff was prepared as described in the literature [5]. According to x-ray measurements the maghemite (Fe-edl- ff) and the Zn-edl-ff particles have a mean diameter of 10 $\mathrm{nm}$ and $6.3 \mathrm{~nm}$, respectively [8].

The maghemite edl-ff modified by the adsorption of the organic acids (glutamic - sample (A) and aspartic - sample (B)) were prepared by the peptization of the ferrite in a solution containing $10^{-2} \mathrm{~mol} / \mathrm{L}$ of acid then the $\mathrm{pH}$ is adjusted with a $\mathrm{NaOH}$ solution to the value of 7.0. After that acetone is added to induce the precipitation of the modified ferrofluid which is then filtered, thoroughly washed with doubly distilled water, ethanol and acetone, and finally dried in air, resulting in a solid powder with magnetic properties. In this case, only the solid samples were investigated. In the case of the MB modified edl-ff we have prepared three samples:

i) Sample (C) was obtained by the peptization of the maghemite edl-ff in a $\mathrm{pH} 2.0$ solution containing MB, forming a solution with magnetic properties. The amount of solid edl-ff (maghemite) and MB used were adjusted to give a solution at $0.2 \mathrm{~mol} / \mathrm{L}$ in maghemite $\left(4.92 \times 10^{15}\right.$ particles $\left./ \mathrm{mL}\right)$ for a monolayer coverage of MB. We have considered the MB area as $10^{-14} \mathrm{~cm}^{2}(1$ $\mathrm{nm}^{2}$ ) and the maghemite particle area as $3.46 \times 10^{-12}$ $\mathrm{cm}^{2}$. The final MB concentration in solution was then estimated to be ca. $2.8 \times 10^{-3} \mathrm{~mol} / \mathrm{L}$.

ii) Sample (D) was prepared by treating a small portion of sample (C) as described above for the organic acid modified edl-ff. During the preparation of sample $\mathrm{D}$, after precipitation of the solid by the addition of acetone we have observed that the acetone solution turned blue, indicating that not all MB molecules were adsorbed on the maghemite surface. Therefore, the MB surface coverage on sample (C)e must be less than a monolayer.

iii) Sample (E) was obtained by re-dispersing a certain amount of sample (D) in a water solution of $\mathrm{pH}$ 2.0 .

Only analytical grade reactants were used in all chemical preparations. The description of the edl-ff modified samples investigated in this work is summarized in Table 1.

Table 1. Summarized description of the maghemite edl-ff modified samples

\begin{tabular}{lc}
\hline Sample & Description \\
& Substrate/modifier-sample condition \\
\hline A & maghemite edl-ff/glutamic acid - powder \\
B & maghemite edl-ff/aspartic acid - powder \\
C & maghemite edl-ff/methylene blue - solution \\
D & maghemite edl-ff/methylene blue - powder \\
E & maghemite edl-ff/methylene blue - redispersed solution \\
\hline
\end{tabular}

The Raman and laser induced fluorescence (LIF) measurements were performed on a Renishaw Raman System 3000, equipped with a high performance CCD camera and an Olympus microscope. An 80x objective was used to focus the laser beam on the sample and to collect the scattered radiation. The Raman system was configured in order we could make use of its confocal capability to avoid the strong re-absorption from the MB samples and the strong fluorescence from the glass capillary walls. The laser excitation sources were the 
$632.8 \mathrm{~nm}$ line from a HeNe laser and the $514.5 \mathrm{~nm}$ line from $\mathrm{Ar}^{+}$laser (Spectra- Physics). The Fourier transform infrared (FTIR) spectra were obtained on Bomem (MB-series) FTIR instrument. The FTIR spectra were obtained from $\mathrm{KBr}$ pellets.

\section{Results and Discussion}

\section{Raman spectroscopy of non modified edl-ff}

Fig. 1 displays Raman spectra of Fe and Zn edl-ff solutions excited at $632.8 \mathrm{~nm}$. While no infrared spectrum can be obtained from these water solutions, the Raman spectra are very reach in information. Note that the Fe edl-ff is quite different from that of $\mathrm{Zn}$ edlff. In a previous work [8] we have performed a detailed investigation of these systems. In both cases, the broad features observed below $700 \mathrm{~cm}^{-1}$ are characteristic of the nanoparticle nuclei as being composed mainly of maghemite $\left(\gamma-\mathrm{Fe}_{2} \mathrm{O}_{3}\right)$. We have identified Raman features characteristic of other species like non stoichiometric Fe(III) oxy-hydroxide (the shoulder near $720 \mathrm{~cm}^{-1}$ ) that is only observed for the Fe-edl-ff.

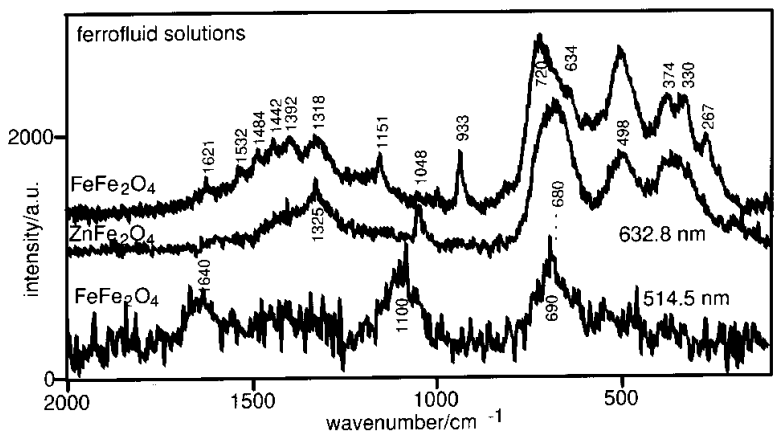

Figure 1. Raman spectra of Fe and Zn edl-ff in solution. The lower spectrum was excited at $514.5 \mathrm{~nm}$.

Changing the laser excitation to the green, a completely different Raman spectrum is observed (see Fig. 1 ). The broad feature near $690 \mathrm{~cm}^{-1}$ is observed only in solution for both $\mathrm{Fe}$ and $\mathrm{Zn}$-edl-ff and was assigned to the presence of $\mathrm{Fe}(\mathrm{OH})_{3}$ in the external surface of the nanoparticle [8]. Another interesting aspect that deserves comments is that all features observed above $950 \mathrm{~cm}^{-1}$ at $632.8 \mathrm{~nm}$ excitation have vanished at 514.5 $\mathrm{nm}$ excitation. Since the Fe-edl-ff solution has an electronic absorption near $650 \mathrm{~nm}$ that is not observed for the Zn-edl-ff, the presence of these features were assigned to a resonant processes involving the density of phonon states in the maghemite ferrofluid (Fe-edl-ff).

The particle diameter is much smaller than the laser wavelength, therefore the momentum conservation rule relaxes, forbidden phonons become allowed and are observed only at $632.8 \mathrm{~nm}$ excitation due to a resonance process, i.e., the electronic transition near $650 \mathrm{~nm}$ gives intensity to the Raman phonons.

\section{Raman spectra of edl-ff modified by amino-acids}

Fig. 2 displays the FTIR and Raman spectra of solid samples of maghemite and maghemite modified by the adsorption of glutamic (sample A) and aspartic (sample B) acids. The broad structure below 800 $\mathrm{cm}^{-1}$ in the FTIR spectrum is due to maghemite vibrations. The Raman signals associated to the maghemite appear as broad features at 360,502 , and $700 \mathrm{~cm}^{-1}$ [8]. The very broad and poor resolved feature near $1400 \mathrm{~cm}^{-1}$ is also characteristic of maghemite [8]. The main characteristic vibrations of the adsorbates, the $\mathrm{NH}$ bending and $\mathrm{C}=\mathrm{O}$ stretching modes are expected to appear above $1400 \mathrm{~cm}^{-1}$. Comparing the FTIR spectra of maghemite and those of modified maghemite one clearly sees features near 1610, 1540 and $1431 \mathrm{~cm}^{-1}$ which could be due to the adsorbates. The signal at $1385 \mathrm{~cm}^{-1}$ could be due to the coadsorbed $\mathrm{NO}_{3}^{-}$ion. On the other hand, FTIR spectra from pure $\mathrm{KBr}$ pellets have also shown this feature. Since the nitrate ion usually presents a strong Raman feature near $1050 \mathrm{~cm}-$ 1 and we have not observed any feature in this region for the non-modified sample (Fig.1a), we believe that this feature is due to some impurity in the $\mathrm{KBr}$ used to prepare the pellets. The Raman signals observed at 1044 and $1650 \mathrm{~cm}^{-1}$ (weak) are due to the CCO and $\mathrm{C}=\mathrm{O}$ stretching modes of glutamate (or aspartate), respectively.

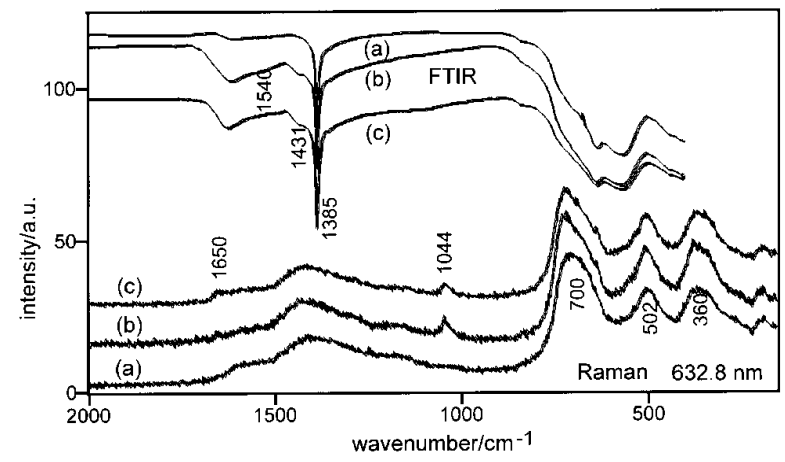

Figure 2. FTIR (KBr pellets) and Raman spectra (powder) of: (a) maghemite edl-ff as solid, (b) glutamic (sample A), and (c) aspartic (sample B) modified maghemites.

Fig. 3 presents the Raman spectra of the maghemite samples in the $\mathrm{CH}$ stretching region. The broad feature near $2944 \mathrm{~cm}^{-1}$ is a further evidence for the adsorption of the organic acids on the maghemite surface. From the above results, it seams that the organic acids are adsorbed on the maghemite surface as carboxylates, probably by forming chelate structures with the Fe(III) ions on the maghemite surface. 


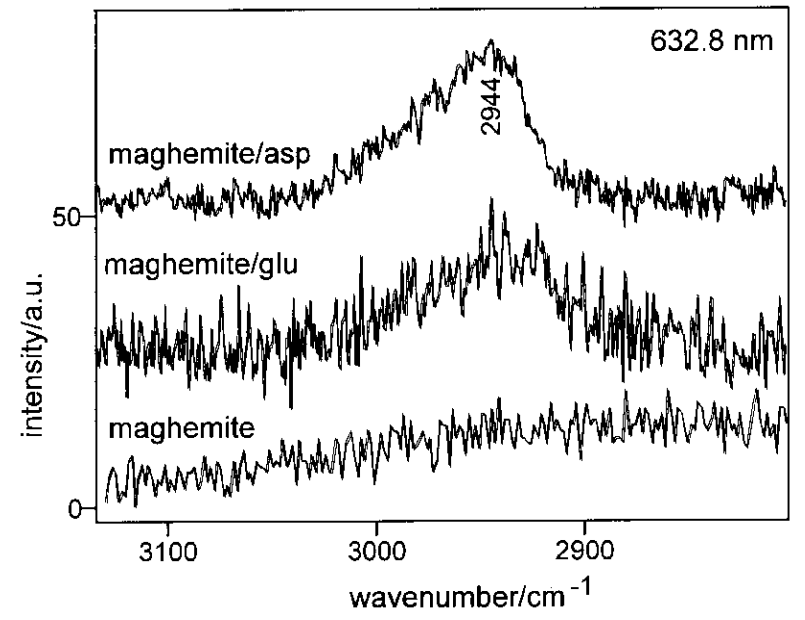

Figure 3. Raman spectra of the indicated samples in the $\mathrm{CH}$ stretching region (asp and glu stand for aspartic and glutamic acid modified maghemites, respectively).

In a previous work [16] we have performed a conductimetric titration of the Fe-edl-ff solution with aspartic and glutamic acids. From these results we were able to drawn Langmuir-type adsorption isotherms and found no evidence for multilayer adsorption for both organic acids. The maghemite particle area is $3.46 \times 10^{-12} \mathrm{~cm}^{2}$ and the adsorbate surface concentration was estimated to be ca. $1.67 \times 10^{-10} \mathrm{~mol} / \mathrm{cm}^{2}$ (we have considered the molecule area as $1 \mathrm{~nm}^{2}$ ). This result and the fact that the FTIR spectra of the modified edl-ff present very weak signals from the adsorbates are very interesting since normal Raman spectroscopy could not explain the observed results. These results call for an explanation based on a resonance process mediated by the particle surface as causing an enhancement of the Raman features due to the adsorbates.

\section{The Raman spectra of Methylene Blue modified maghemite}

Methylene Blue is a strongly absorbing dye. Even at low concentrations $\mathrm{MB}$ is present in the form of dimer and monomer. The dimer absorbs at $610 \mathrm{~nm}(\epsilon=$ $\left.9.06 \times 10^{4} \mathrm{~L} \cdot \mathrm{mol}^{-1} \cdot \mathrm{cm}^{-1}\right)$ and the monomer at $662 \mathrm{~nm}$ $\left(\epsilon=3.88 \times 10^{4} \mathrm{~L} \cdot \mathrm{mol}^{-1} . \mathrm{cm}^{-1}\right)[17]$. At $10^{-4} \mathrm{~mol} / \mathrm{L} \mathrm{MB}$ concentration and $\mathrm{pH} 2.0$, the dimer absorption appear at $608 \mathrm{~nm}$ and is stronger than that of the monomer at $663 \mathrm{~nm}$. Therefore, Raman spectra excited at $632 \mathrm{~nm}$ are resonance enhanced either for dimer and monomer MB species. It is worth to mention that there is no report on the literature on the Raman spectrum of MB in solution at the resonance condition, i.e., using a red laser line.

Fig. 4 shows FTIR and resonance Raman spectra (excited at $632.8 \mathrm{~nm}$ ) of maghemite edl-ff that was modified by the adsorption of MB (samples C and D). We did not observe any signal in the FTIR spectrum of the solid sample that could be characteristic of MB. Comparison of this spectrum with that of pure maghemite edl-ff reveals that the feature near $1385 \mathrm{~cm}^{-1}$ (characteristic of the presence of $\mathrm{NO}_{3}^{-}$ion) becomes broader suggesting that the MB molecule, a cation, interacts with the adsorbed nitrate by ion pair formation.

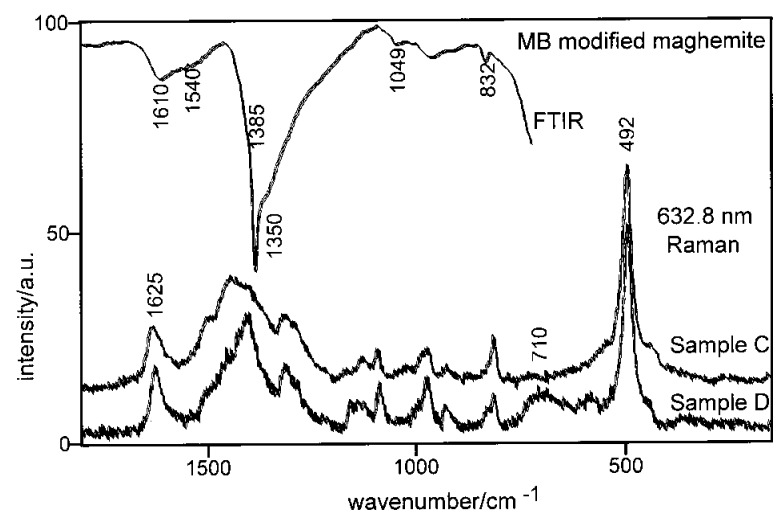

Figure 4. FTIR (Sample D in KBr pellet) and Raman spectra of MB modified maghemite samples $\mathrm{C}$ and $\mathrm{D}$ at 632.8 nm excitation.

Contrary to the FTIR spectrum, the resonance Raman spectra of the MB modified edl-ff solution and solid (samples $\mathrm{C}$ and $\mathrm{D}$, respectively) present several features that are characteristic of MB. In the case of the solid sample, note that the presence of a broad feature near $710 \mathrm{~cm}^{-1}$ characterizes the maghemite nanoparticle and the features at 492 and $1625 \mathrm{~cm}^{-1}$ are characteristic of the adsorbed MB. As we mentioned in the experimental part, sample $\mathrm{C}$ has MB molecules in solution. However, one does not observe any signal that could be assigned to MB in solution. The comparison was done with the Raman spectrum of MB in solutions excited at pre-resonance condition, at $514.5 \mathrm{~nm}$ excitation [18].

It is well known that molecules adsorbed on a roughened silver electrode can present the surface enhanced Raman scattering (SERS) effect [21-23]. In this case, surface plasmon-polariton resonances and photon assisted (surface mediated) charge transfer excitation are responsible for the enhancement of the Raman spectra [21-23]. The enhancement factor for SERS can be as large as $10^{6}$. If the adsorbate itself is a chromophore that strongly absorbs the laser excitation used in the experiment one can have the surface-enhanced resonance Raman scattering (SERRS) effect. At these conditions, the enhancement factor can be $10^{9}$ or even larger [22,24]. For comparison purposes, Fig. 5 displays the resonance Raman spectrum of solid MB and the SERR (surface-enhanced resonance Raman) spectra of MB adsorbed on a silver electrode. The Raman spectrum of MB in solution, where it is present as a dimer is very similar to the solid state spectrum. The main characteristic features of the aggregated MB molecules are the Raman signals at 450 and $502 \mathrm{~cm}^{-1}$. When MB adsorbs on the silver electrode at monolayer condition [23], a new feature emerges at $485 \mathrm{~cm}^{-1}$ while those at 
450 and $502 \mathrm{~cm}^{-1}$ are very weak.

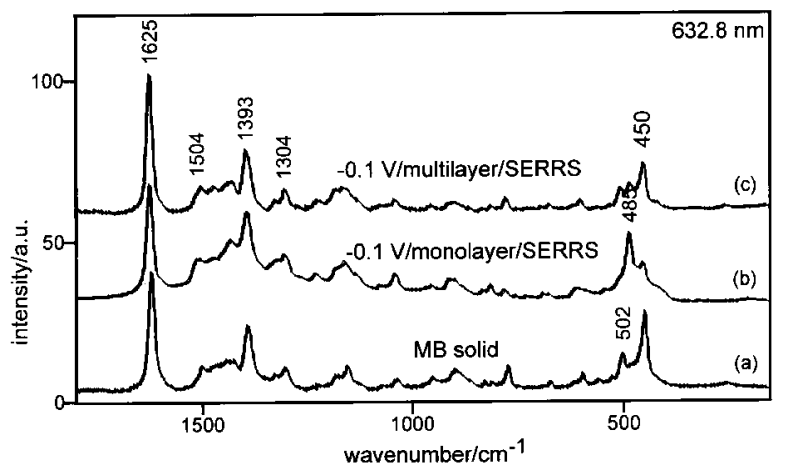

Figure 5. Raman spectrum of solid MB (a) and SERR spectra of MB adsorbed on a silver electrode at monolayer (b) and multilayer conditions (c).

On the other hand, the SERR spectrum at multilayer condition [23] presents the Raman features at 450 and $502 \mathrm{~cm}^{-1}$ relatively stronger than that at 485 $\mathrm{cm}^{-1}$, suggesting that the number of aggregated MB molecules at the electrode surface has increased.

Comparing the RR spectra of Fig. 4 and those of Fig. 5 we can also see that Raman signals due to MB adsorbed on the maghemite surface are broader than those of solid MB or adsorbed on a silver electrode. For instance, the feature at $1625 \mathrm{~cm}^{-1}$ has a full width at half maximum (fwhm) value of $38 \mathrm{~cm}^{-1}$ when MB is adsorbed on the edl-ff while in the spectra of Fig. 5 the fwhm value for this Raman signal is $17 \mathrm{~cm}^{-1}$.

From the above results one can conclude that the MB molecule adsorbs on the maghemite edl-ff nanoparticle surface as a monomer. The adsorbed monomer has a Raman signal that is stronger than that of MB present in solution and that the surface heterogeneity in this case is larger than that of the silver electrode.

We have also obtained Raman spectra of the MB modified edl-ff excited at $514.5 \mathrm{~nm}$. Almost the same Raman lines observed at $632.8 \mathrm{~nm}$ are observed at 514.5 $\mathrm{nm}$ excitation, but the relative intensities are quite different (see Fig. 6). As above mentioned, the MB dimer in solution absorbs near $610 \mathrm{~nm}$. This means that any interaction of MB has the effect of increasing the energy of the $\pi-\pi^{*}$ transition responsible for the blue color of MB. The UV-vis absorption spectrum of the MB modified edl-ff presents a strong absorption near $400 \mathrm{~nm}$. As a consequence one needs to dilute the working solution and use a very thin cuvette. The absorption spectra obtained in this way reveal a not well defined feature near $640 \mathrm{~nm}$. On the other hand, the visible absorption spectrum of a pure Fe-edl-ff solution also presents a feature near $650 \mathrm{~nm}$. Therefore it is difficult to assign the $640 \mathrm{~nm}$ feature to the adsorbed MB.

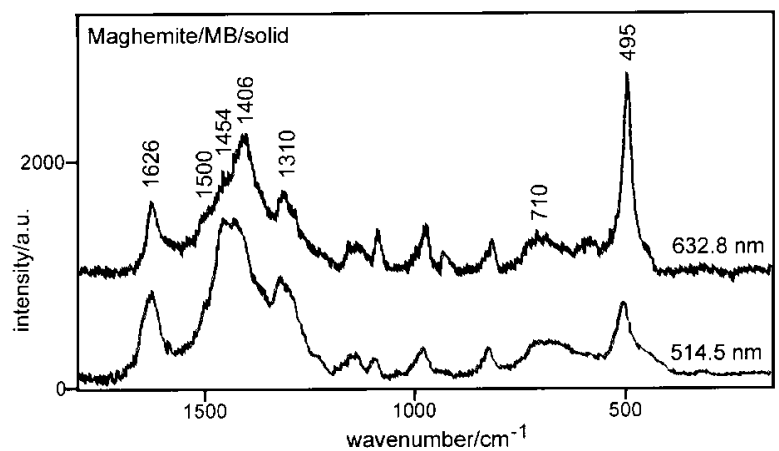

Figure 6. Raman spectra of the MB modified maghemite edl-ff as a solid powder (sample D) at the indicated laser excitations.

The RR spectra of the MB solution [20] show that as the exciting energy approaches resonance, all observed vibrational modes get enhanced, indicating that the electronic $\pi$ system is delocalized over all bonds in the rings, i.e., there is no localized chromophore. On the other hand, the spectra displayed in Fig. 6 shows that the MB vibrational modes at 495 and $1454 \mathrm{~cm}^{-1}$ are preferentially enhanced on going from 514.5 to 632.8 $\mathrm{nm}$ excitation. This is an indication that the surface of the maghemite particle has induced some changes on the electronic delocalization of the $\pi$ system. Further evidences for this perturbation was obtained from laser induced fluorescence (LIF) measurements.

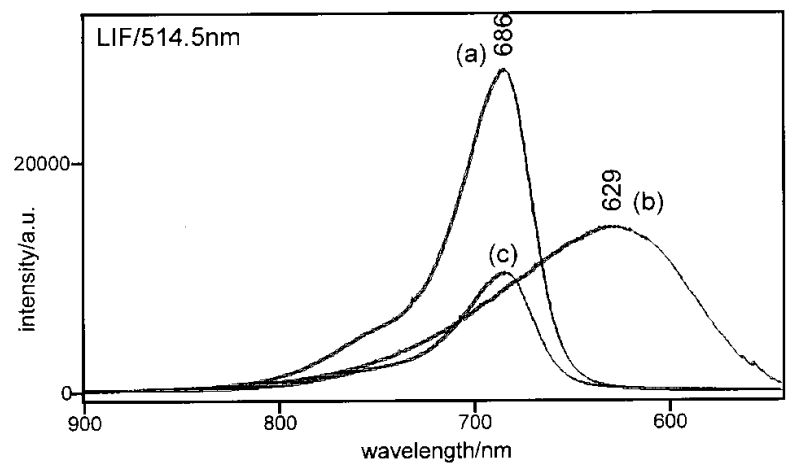

Figure 7. LIF spectra of the investigated MB modified maghemite edl-ff: (a) Sample C; (b) Sample D; (c) Sample E.

Fig. 7 displays the LIF spectra of samples $\mathrm{C}, \mathrm{D}$, and E. Since we have MB in solution in the case of sample C, its LIF spectrum is identical to that of MB in solution. The LIF spectrum of sample $\mathrm{D}$, as a solid powder, shows that the emission maximum has shifted ca. 57 $\mathrm{nm}$ to higher energy (a blue shift). This means that the emitting electronic state is at a higher energy level when MB is adsorbed on the maghemite surface. This result is in perfect agreement to the above conclusion based on the RR measurements, that the electronic $\pi$ system of the MB molecule is affected by the interaction with the maghemite surface.

As mentioned in the experimental part, sample $\mathrm{C}$ has MB in solution. Despite of that no Raman signal 
from $\mathrm{MB}$ in solution was observed at pre-resonance or resonance. Furthermore, no signal from adsorbed MB was observed in the FTIR spectrum. These results together suggest that the Raman cross section for MB adsorbed on the maghemite surface is larger than that of $\mathrm{MB}$ in solution or even larger than the cross section for infrared absorptions, which usually are $10^{8}$ to $10^{10}$ larger than Raman cross sections [24]. Therefore, as in the previous cases, we believe that some kind of surface enhanced phenomena is playing a rule in the Raman spectra of MB-modified maghemite ferrofluid.

\section{Final remarks}

The results presented in this work have clearly shown that Raman spectroscopy is a powerful technique in the characterization of pure magnetic fluids and those modified by the adsorption of molecules with different chemical properties as aminoacids and photosensitive species.

In the case of maghemites we have shown that by changing the laser excitation energy it is possible to tune on and off the resonance process for different species present in the ferrite nanoparticles. For instance, at red excitation one observes the main contribution from maghemite $\left(\gamma-\mathrm{Fe}_{2} \mathrm{O}_{3}\right)$ and non stoichiometric Fe(III) oxy-hydroxides. At green excitation one observes the main contribution of the $\mathrm{Fe}(\mathrm{OH})_{3}$ at the nanoparticle surface.

This is a very interesting aspect since with the use of fiber optics one can monitor the preparation of ferrofluids by in situ Raman spectroscopy.

In the study of the adsorption of glutamate and aspartate it was demonstrated that these species adsorb chemically on the maghemite surface by forming chelate compounds with the $\mathrm{Fe}(\mathrm{III})$ ions present on the maghemite surface.

The investigation of the MB modified maghemites have shown that Methylene blue, a cation, adsorbs as a monomer on the maghemite surface, probably by ion pair formation with the coadsorbed $\mathrm{NO}_{3}^{-}$ions. A remarkable aspect that deserves comment is the observation that the MB-maghemite ferrofluid is still strongly fluorescent. This result suggests that the MB-modified maghemite may be a good candidate for laser photodynamic therapy. In this sense, further investigations are necessary in order to determine the quantum efficiency of the photoluminescence and the quantum efficiency of triplet state generation, which are very important in the ${ }^{1} \mathrm{O}_{2}$ generation.

Finally, a resonant process involving the nanoparticle and the species at the nanoparticle surface must be present to explain the facts that almost no signal from the investigated adsorbates are observed in FTIR spectra of the modified maghemites. This aspect deserves further and more quantitative investigations in order one can estimate the enhancement factors.

\section{Acknowledgements}

The authors gratefully acknowledge the Laboratório de Espectroscopia Molecular do Instituto de Química da Universidade de São Paulo (where the Raman measurements were performed), and $\mathrm{CNPq}$ for research fellowships and financial support.

\section{References}

[1] M.S. Brandt and M. Stutzmann,. Solid State Commun. 93, 473 (1995).

[2] J.L. Gole, F. Dudel, D.R. Grantier and D.A. Dixon, Phys. Rev. B. 36, 2137 (1997).

[3] F. Dudel, J.L. Gole, M. Reiger, P. Kohl, J. Pickering and L. Bottomley, J. Electrochem. Soc. 143, L-164 (1996).

[4] F.A. Tourinho, R. Franck and R. Massart, J. Mater Sci. 25, 3249 (1990).

[5] F.A. Tourinho, J. Depeyrot, G.J. da Silva, and M.C.L. Lara, Braz. J. Phys. 28, 413 (1998).

[6] A. Hugot-Le Goff, S. Joiret, and P. Falaras, J. Phys. Chem. B, 103, 9569 (1999).

[7] N. Esser, Appl. Phys. A-Matter, 69, 507, (1999).

[8] M.H. Sousa, F.A. Tourinho and J.C. Rubim, J. Raman Spectrosc. 31, 185 (2000).

[9] E.J. Heller, Acc. Chem. Res. 14, 368, (1981).

[10] D. Tannor and E.J. Heller, J. Chem. Phys. 77, 202 (1982).

[11] E.J. Heller, R.L. Sundberg and D. Tannor, J. Phys. Chem. 86, 1822 (1982).

[12] U. Häfeli, W. Schüt, J. Teller and M. Zborowski, Eds. Scientific and Clinical Applications of Magnetic Carriers, Plenum Press: New York, 1997. U. Häfeli and M. Zborowski, Eds. Proc. $2^{\text {nd }}$ International Conference on the Scientific and Clinical Applications of Magnetic Carriers, J. Magn. Magn. Mater. 194 (1999).

[13] E.M. Tutite and J.M. Kelly, J. Photochem. Photobiol. B, 21, 103 (1993).

[14] J.M. Fernandez, M.D. Bilgim and L.I. Grossweiner, J. Photochem. Photobiol. B, 37, 131 (1997).

[15] C.H. Sibata, V.C. Colussi, N.L. Oleinck, and T.J. Kinsella. Braz. J. Medical and Biological Research. 33, 869 (2000).

[16] M.H. Sousa, J.C. Rubim and F.A. Tourinho, J. Magn Magn. Mater. 225, 67 (2001).

[17] P.C. Yates and B. Patel, J. Molec. Struct. (Theochem), 315, 117 (1994).

[18] S.H.A. Nicolai. Ph.D Thesis, Instituto de Química da USP, (1998).

[19] D.L. Jeanmaire and R.P. van Duyne, J. Electroanal. Chem. 84, 1 (1977).

[20] A. Otto, I. Mrozek, H. Grabhorn and W. Akemann, J. Phys. Condens. Matter, 4, 1143 (1992). 
[21] J.C. Rubim, P. Corio, M.C.C. Ribeiro and M. Matz, J. Phys. Chem. 99, 15765 (1995).

[22] P. Corio, J.C. Rubim and R. Aroca, Langmuir, 14, 4162 (1998).

[23] S.H.A. Nicolai and J.C. Rubim, (1996), private communication.
[24] W.B. Person, Infrared Intensities and Atomic Polar Tensors, Ch.4 in Vibrational Intensities in Infrared and Raman Spectroscopy, (Studies in Physical and Theoretical Chemistry, 20) ed. W.B. Person and G. Zerbi, Elsevier Sci. Publ. Comp., Amsterdan (1982). 